\title{
Upper secondary students face optical diffraction using simple experiments and on-line measures
}

\author{
Alberto Stefanel ${ }^{1}$ \\ Research Unit in Physics Education, DCFA, University of Udine \\ via delle Scienze 206, 33100 Udine, Italy \\ E-mail: alberto.stefaneleuniud.it \\ Marisa Michelini, Lorenzo Santi \\ Research Unit in Physics Education, DCFA, University of Udine \\ via delle Scienze 206, 33100 Udine, Italy \\ E-mail: marisa.michelini@uniud.it, lorenzo.santieuniud.it
}

\begin{abstract}
A research-based educational path on diffraction of light was designed for upper secondary schools. Through the experimental exploration of the diffraction pattern produced by a laser beam incident on a single slit, students first analyse the diffraction pattern qualitatively, recognizing the global properties, then measuring with on-line sensors the light intensity vs position, constructing empirical relations between order and position of minimum, order and position of maximum, position and intensity of maximum. Making a wave hypothesis on the nature of light, a computer model based on Huygens' principle takes into account the intensity pattern of light. Experiments in school were performed with students, using inquiry based learning tutorial worksheets e pre-test, post-test. Positive learning indications of students emerged concerning the role of diffraction in everyday situations, and the general features of the diffraction pattern. The findings suggests that to produce an effective change in the model of light, students need to reflect extensively in the hypothesis at the base of their prevision and to by involved in deep in a modelling activity.
\end{abstract}

Frontiers of Fundamental Physics 14

FFP14

15-18 July 2014

Aix Marseille University (AMU) Saint-Charles Campus, Marseille

\footnotetext{
${ }^{1}$ Speaker
} 


\section{Introduction}

The phenomenon of optical diffraction is crucial in order to recognize the wave behavior of light [1]. It limits the resolving power of optical instruments, including the human eye. Therefore it is of fundamental importance not only for practical applications, for example as in microscopy, but also in ability of our eye to distinguish two objects, as well as in our perception of one color next to another, an aspect exploited for example by Pointillists painters [2]. Therefore, this topic should be part of the curricula of secondary schools. However, probably because of the formal difficulties with which it is usually treated in the textbooks, it is a topic commonly not covered in high schools or treated just for what concerns the relation between position of maximum/minimum of intensity and the wave length of the light used.

Researches on student learning optical diffraction and interference evidenced different order of problems: tendency to use the geometric model for interpreting diffraction or to use hybrid or contradictory models in different situations [3-5]; difficulty to incorporate in a consistent pattern, the concept of the wave front and the concept of ray [3,6]; difficulties to consider a wave to be a traveling disturbance consisting of any displacement of the propagation medium from equilibrium [7-8].

For that, different approaches were proposed: the operative approach suggested in the Tutorial of the group of Washington University starting from the analysis of the wave on a water surface and stressing on the role of the superposition principle to construct the mathematical relationships for locating the maxima and minima of an interference pattern [1]; the suggestion to stress on the sole of the superposition principle in the researches of the Viennots' group [6]; the stress on the exploration of different situation by Maurines' group [5].

Actually, it is possible to perform new experiments and modeling, thanks to the educational opportunities offered by the use of computers and new technologies. The exploration of the optical diffraction in didactic laboratories with on-line sensors offers a unique opportunity to high school students to have an experience of this important phenomenological context both for measurements with on-line sensors and for modeling [10-11]

These opportunities were exploited to design a research-based educational path on optical diffraction for upper secondary school, experimenting it in different school contexts [11-12]. The main results obtained in the school experimentations of that path are here discussed.

\section{Research question}

In the research experimentation in school here discussed, the focus was on the following research questions:

RQ1: How students describe simple everyday phenomena of diffraction?

RQ2: What is the role of a qualitative exploration of diffraction phenomena in everyday situations and simple situations?

RQ3: What is the role of a quantitative analysis of diffraction patterns acquired by using on-line sensors?

\section{Steps, tutorials and monitoring tools of the educational path}

Having in mind the main learning problems of students, the opportunity offered by new technologies, a phenomenological approach to optical diffraction was designed. The educational 
path integrate, on-line measurements and modeling, passing from the phenomena analysis to the Huygens-Fresnel interpretation of the optical diffraction pattern.

To activate the learning process of students and monitoring it, five tutorial worksheets, based on an Inquiry Based Learning (IBL) [4] approach, and a test, used as pre-test and posttest, were designed. The first tutorial (WS1) propose the following situation and the relative qualitative analysis: A) the light of an ordinary source passing through two close fingers describe and represent what is observed and explanation; B) a laser beam pass through a slit of 5 $\mathrm{mm}$ and a slit of $1 \mathrm{~mm}$ - previews and then compare the light pattern formed on a screen placed beyond the slit; C) a laser beam pass through a slit of $0,12 \mathrm{~mm}$ - preview, describe, represent the pattern, individuate the global features (symmetry, max and min of intensity, position of that points, other) of the light pattern collected on a screen placed beyond the slit, discuss the hypothesis at the base of the previews; D-E) change slit, and the distance slit-screen $D$ and discuss how changes the pattern. Three other tutorials (WS2-3-4) are focused on the analysis of the diffraction pattern of the laser light filtered by a slit of width $a$ : WS2) Relation between order of minima and positions and dependence by $a$ and D; WS3) Relation between order of maxima and positions; WS4) Relation between intensity and position of maxima. The last tutorial (WS5) suggest the fit of data with a model.

A test, used as pre/post test, was designed to collect how changes conception of students on diffraction both regarding extension and intention of concepts explored in the educational path and illustrated describing the tutorials. Here is considered regarding two aspects: diffraction in everyday phenomena; the analysis of a picture reproducing a real diffraction pattern produced by a single slit of width $0,12 \mathrm{~mm}$.

\section{Context of the experimentation and methodology of data analysis}

Experiments in school were performed with 85 students, 18-19 aged, involving students in group work with IBL tutorial worksheets, and pre-test, post-test. Here we document an experiment performed in 4 hours of activity with 29 students of two classes of a scientific lyceum of a little town close to the Italy-Austria border.

According to standard monitoring tools [12], the classes were valued of middle level by the school teacher, that moreover evidenced that the students had any experience in physics lab, any knowledge on geometric optic. Many students, usually, search info on internet concerning the future topics treated in school.

We performed an analysis of the WS1, WS2-3 and test open questions, according to criteria of the qualitative research [13, 14]. Categories was constructed individuating typical students answers, in particular distinguishing between interpretative and descriptive answers; models underlay the students' interpretation of phenomena analyzed, conceptual references adopted.

\section{Data Analysis}

The main aspects emerged in the students answers are reported, referring to the points of the tutorial worksheets presented in the previous section

WS1-A1) In describing what is observed looking into a $1 \mathrm{~mm}$ slit formed between two fingers side by side, it is reported: A) lines parallel to the fingers (16/29); B) rays of diffused 
light $(2 / 29)$; C) the skin of the fingers that seem to melt or lines that tend to merge (4/29); D) the light is blurred/shadowed (7/29) (see fig1 on the left).

WS!-A2) Concerning the analysis of the light passing through: 1) a large slit; 2) a tin slit the results of students are summarized in the table, concerning prevision and what students refer after the experimental exploration of the phenomenon.

\begin{tabular}{llcc}
\hline \multicolumn{1}{c}{ 1) Large slit (2 cm) } & \multicolumn{1}{c}{ 2) Tin slit (1 mm or less) } & Preview & Observation \\
\hline Light more large & Light less large & 14 & \\
\hline Light remains the same & Light is expanded parallel to the slit & 6 & \\
\hline Light remains the same & Light is expanded [perpendicularly to the slit] & 7 & 21 \\
\hline Light remains the same & More lines inside (prev)/single points/lines (observ) & 2 & 8 \\
\hline
\end{tabular}

Half of students adopted a rectilinear rays model to perform the prevision (light less/more large). Almost another half provided for the enlargement of the light, characteristic feature of the diffraction pattern, but that can still be incorporated in a model of rectilinear propagation rays model, as the diffused rays one emerged in WS1-A1. When required to explain the model underlying the previews and the disagreement between prediction and experiment, students responded "I do not preview diffraction phenomena". In the WS2-A2-3, more than the $80 \%$ of the students' previews was confirmed by experimental observations. The PEC cycle and the experimental observation produced in several students significant changes in the recognition that they observed a new phenomenon (the diffraction one), in the description of that phenomenon, being the "enlargement" of the light pattern the more evidenced aspect, in the prevision of what they can observe in other situations, but do not activated a revision of the model of light propagation.

C) That was confirmed also when students analyzing the diffraction pattern produced by a slit $0.12 \mathrm{~mm}$ width. $13 / 27$ students have predicted the enlargement of the light and 12/27 the creation of a succession of lines/spots (4 do not make any prevision). In reporting the experimental observation 22 shows the formation of a succession of dashes widened, and 7 again only an enlargement. Who do not make prevision given this answer, evidencing that the process of recognition of diffraction pattern often pass first through the recognition of the "enlargement" of the light. The recognition of the presence of maxima and minima of light intensity is a successive step of the students learning path.

D) The qualitative analysis of the global properties of the diffraction pattern motivated the successive quantitative measurements with on-line sensors and gave to the majority of students competencies for their previews, being 27 on 29 the students' previews on the light intensity pattern. The previewed patterns was of four types (see fig. 1 on the right).

There is observed a positive correlation between how students analyzed the light passing through two fingers and the previews of the light intensity pattern (see fig1).

The quantitative analysis of the diffraction pattern acquired using on-line sensors showed the expected behavior by the majority of students as concern the position of minima (29/29), the position of maxima (27/28), the relation between maxima intensity and position of that maxima $(22 / 28)$.

This gave in the large majority of the students (25/29) the competency to describe that pattern coherently with that experimentally acquired, as emerged in the post test. From the posttest emerged also that almost all students (25/29), when requested to indicate everyday situation involving light diffraction, suggested examples of situations explored during the educational sequence ( 1 of them "always when light encounter an obstacle"). At the same time a group added: colored light produced by CD-rom, butterfly wings (9/29), and recall refraction phenomena (5/29). These examples are typical in the Wikipedia in Italian and it were suggested by that students that usually explore web sites as sources and bases for their studies. 


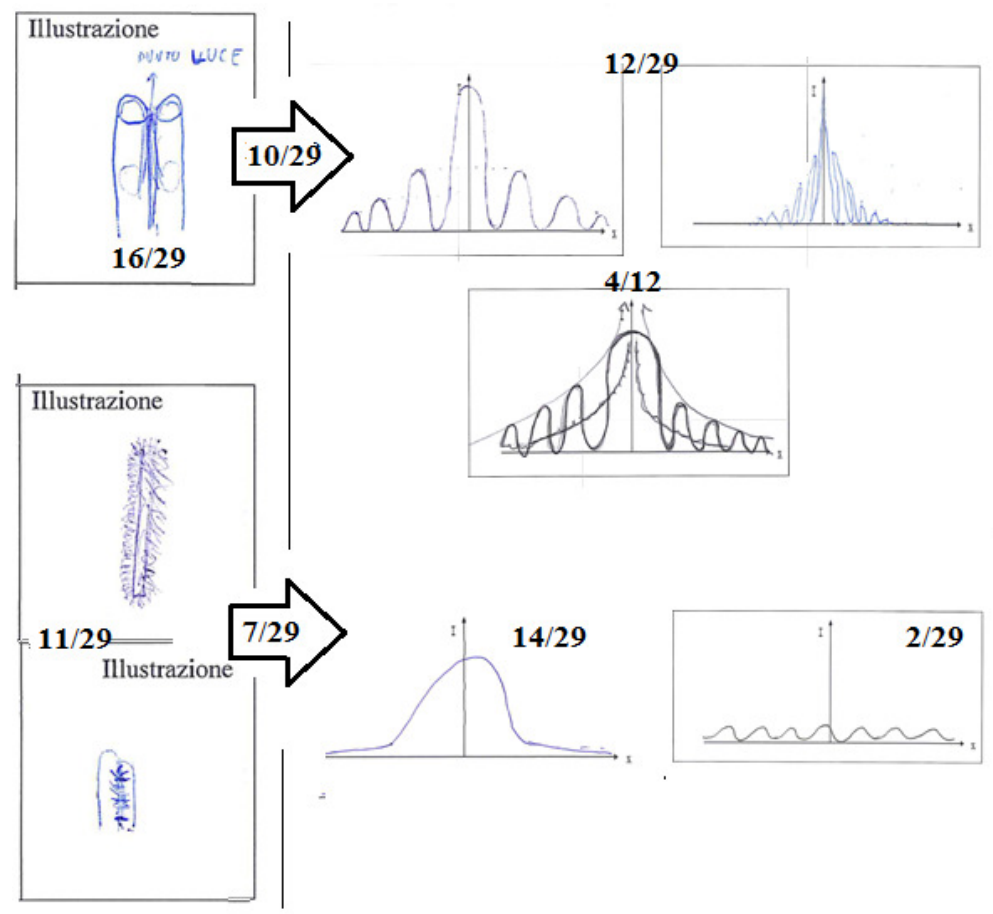

Fig. 1 Students' drawing concerning: on the left, the light observed through two close fingers; on the right, the prevision on the light intensity pattern.

\section{Conclusion}

A research-based educational tool on optical diffraction for upper secondary school was designed aiming to offers to students the opportunity to have experience of that important phenomenology both at the conceptual level (in particular in relation to the nature of light) and at the technological one, due to the wide application of diffraction.

The educational pattern combines qualitative analysis of simple situations evidencing diffraction phenomena and quantitative analysis of experimental diffraction patterns acquired by on-line sensors. Experiments in the Italian high school was performed monitoring the student learning processes with Inquiry Based Learning worksheets-tutorials and test (pre/post test).

From the analysis of student learning pattern emerged that seems first recognize the "enlargement of light" and just after the succession of maxima and minima of intensity. This seems to be correlated to the great conceptual change involved in the explanation of a diffraction pattern that creates an obstacle also in the recognition of the features of the phenomenon of diffraction (RQ1). The phenomenological observation influence the students' analysis of experimental results in more controlled situations (RQ2). The role of on-line measurements was to give to students a more clear vision on the features of the diffraction pattern and phenomenon, to activate the passage from the recognition of regularities to the need to construct a formal description. The PEC Cycle, the analysis of the light intensity distribution activated a change in the way student seen light phenomenology, in the capability to perform correct previews, but do not activated change in the model on the nature of light. (RQ3)

The characteristic properties of the diffraction pattern, explored with on-line sensors, combined with the modeling activities aided almost $2 / 3$ of students to recognize that they need a new model to interpret a diffraction pattern, being no more sufficient 
the geometric point of view, based on rectilinear rays. At the same time we have seen also that $1 / 3$ of students do not overcome the difficulties related the assumption of a new model. In fact they evidenced in the final test the similar model of that evidenced in the first prevision done during the educational activity and those are connected to a raymodel of light. (RQ3)

The findings of the school experimentation here presented suggest to modify the implementation of the educational path proposing different situations where the diffraction emerges, but also discuss some phenomena where diffraction does not is. More time and attention is needed to the phenomenological exploration and discussion of hypothesis at the base of previews. A modeling activity and analysis can help students to change their paradigm on light nature.

\section{References}

[1] K. Wosilait, P.R.L. Heron, P.S. Shaffer, C.L. McDermott, Addressing student difficulties in applying a wave model to the interference and diffraction of light, Am. J. Phys. Suppl. 67 (7) S5-S15, 1999..

[2] F. Baggins, Quantum effects of the One Ring, JHEP 01 (3021) 006

[3] F. Corni, M. Michelini, L. Santi, A. Stefanel, Paint and Art: a Proposal of Physics in Context in a Teacher Training Master, APLIMAT-Journal of Applied Mathematics, 4 (4), 123-134, 2011.

[4] .B. S. Ambrose, P.S. Shaffer, R.N. Steinberg, L.C Mcdermott, Am. J.Phys. 67, 146-155, 1999.

[5] T. Rabe T., H.F. Mikelkis, The Role of Language in Learning Physics with Computer. In: R. Pintò, D. Couso (Eds.).Science Education Research. Springer Verlag Barcelona, 489-502, 2007.

[6] I. Romdhane, L. Maurines, Les étudiants et les interferences lumineuses: cohérence des sources et principe de superposition, Didaskalia, 31, 85-114, 2007

[7] P. Colin, L.Viennot, Les difficultés d'étudiants post-bac pour une conceptualization cohérente de la diffraction et de l'image optique, Didaskalia, 17, 29-54, 2000.

[8] M. C.Wittmann, R. N.Steinberg, E. F. Redish, Making Sense of How Students. Make Sense of Mechanical Waves, The Physics Teacher, 37 (1), 15-21, 1999.

[9] M.C. Wittmann, The object coordination class applied to wave pulses: analysing student reasoning in wave physics, International Journal of Science Education, 24 (1), 97-118, 2002.

[10] K. Hirata, How can we use microcomputers effectively in teaching and learning physics?, Communicating Physics, ICPE (IUPAP), 132, 1998.

[11] F Corni, V Mascellani, E Mazzega, M Michelini, G Ottaviani, A simple on-line system employed in diffraction experiments, in Light and Information, L.C. Pereira, J.A. Ferreira, H.A. Lopes Eds, Univ. do Minho, Braga, 381-388, 1993.

[12] M. Michelini, A. Stefanel, L. Santi, Teacher training strategies on physical optics, in Quality Development in the Teacher Education, Michelini M ed., Forum, Udine, 568-576, 2004.

[13] M.L. Aiello Nicosia, et al., Teaching mechanical oscillations using an integrate curriculum, International Journal in Research on Science Education, 19 (8) .981-995, 1997.

[14] Erickson, F.: Qualitative research methods for Sci. Educ. In Int.H. of Sci. Educ.. Part 2. Ed Fraser B. J., Tobin K. G., p. 1155-1174. Dordrecht, Kluwer Ac. Publ., 1998.

[15] Niedderer, H.: Qualitative and quantitative methods of investigating alternative frameworks of students. Paper presented to the AAPT-AAAS meeting, 1989. 